\title{
Creating and Deploying an Application with (R)Excel and $R$
}

\section{Thomas Baier, Erich Neuwirth and Michele De Meo}

\begin{abstract}
We present some ways of using $\mathrm{R}$ in Excel and build an example application using the package rpart. Starting with simple interactive use of rpart in Excel, we eventually package the code into an Excel-based application, hiding all details (including $\mathrm{R}$ itself) from the end user. In the end, our application implements a serviceoriented architecture (SOA) with a clean separation of presentation and computation layer.
\end{abstract}

\section{Motivation}

Building an application for end users is a very challenging goal. Building a statistics application normally involves three different roles: application developer, statistician, and user. Often, statisticians are programmers too, but are only (or mostly) familiar with statistical programming (languages) and definitely are not experts in creating rich user interfaces/applications for (casual) users.

For many-maybe even for most—applications of statistics, Microsoft Excel is used as the primary user interface. Users are familiar with performing simple computations using the spreadsheet and can easily format the result of the analyses for printing or inclusion in reports or presentations. Unfortunately, Excel does not provide support for doing more complex computations and analyses and also has documented weaknesses for certain numerical calculations.

Statisticians know a solution for this problem, and this solution is called R. R is a very powerful programming language for statistics with lots of methods from different statistical areas implemented in various packages by thousands of contributors. But unfortunately, R's user interface is not what everyday users of statistics in business expect.

The following sections will show a very simple approach allowing a statistician to develop an easyto-use and maintainable end-user application. Our example will make use of $R$ and the package rpart and the resulting application will completely hide the complexity and the $\mathrm{R}$ user interface from the user.

rpart implements recursive partitioning and regression trees. These methods have become powerful tools for analyzing complex data structures and have been employed in the most varied fields: CRM, financial risk management, insurance, pharmaceuticals and so on (for example, see: Altman (2002), Hastie et al. (2009), Zhang and Singer (1999)).

The main reason for the wide distribution of treebased methods is their simplicity and intuitiveness.
Prediction of a quantitative or categorical variable, is done through a tree structure, which even nonprofessionals can read and understand easily. The application of a computationally complex algorithm thus results in an intuitive and easy to use tool. Prediction of a categorical variable is performed by a classification tree, while the term regression tree is used for the estimation of a quantitative variable.

Our application will be built for Microsoft Excel and will make use of $R$ and rpart to implement the functionality. We have chosen Excel as the primary tool for performing the analysis because of various advantages:

- Excel has a familiar and easy-to-use user interface.

- Excel is already installed on most of the workstations in the industries we mentioned.

- In many cases, data collection has been performed using Excel, so using Excel for the analysis seems to be the logical choice.

- Excel provides many features to allow a high-quality presentation of the results. Preconfigured presentation options can easily adapted even by the casual user.

- Output data (mostly graphical or tabular presentation of the results) can easily be used in further processing - e.g., embedded in PowerPoint slides or Word documents using OLE (a subset of COM, as in Microsoft Corporation and Digital Equipment Corporation (1995)).

We are using $\mathrm{R}$ for the following reasons:

- One cannot rely on Microsoft Excel's numerical and statistical functions (they do not even give the same results when run in different versions of Excel). See McCullough and Wilson (2002) for more information.

- We are re-using an already existing, tested and proven package for doing the statistics.

- Statistical programmers often use R for performing statistical analysis and implementing functions.

Our goal for creating an RExcel-based application is to enable any user to be able to perform the computations and use the results without any special knowledge of R (or even of RExcel). See Figure 1 for an example of the application's user interface. The results of running the application are shown in Figure 2. 


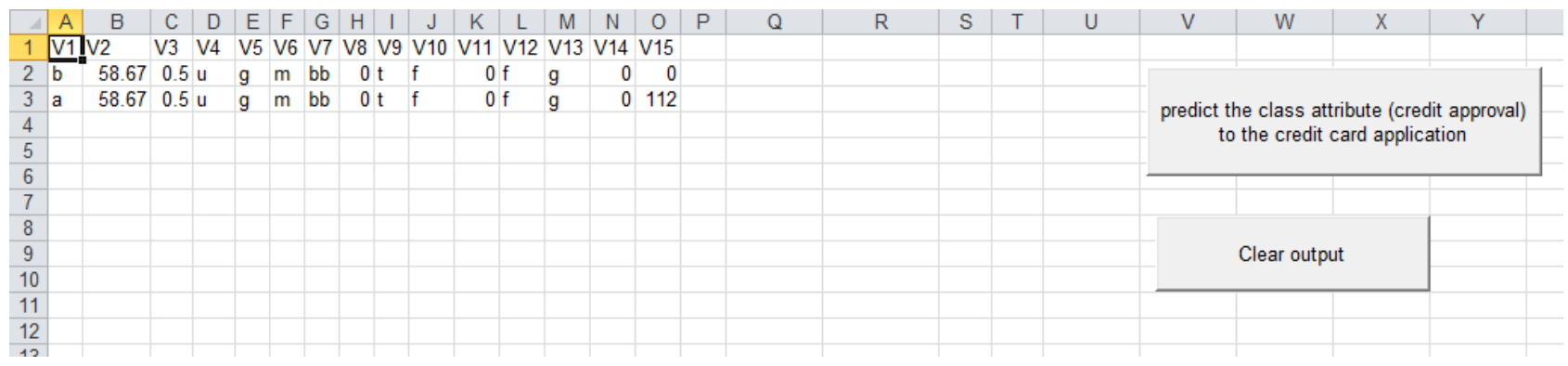

Figure 1: Basic user interface of the RExcel based end-user application.

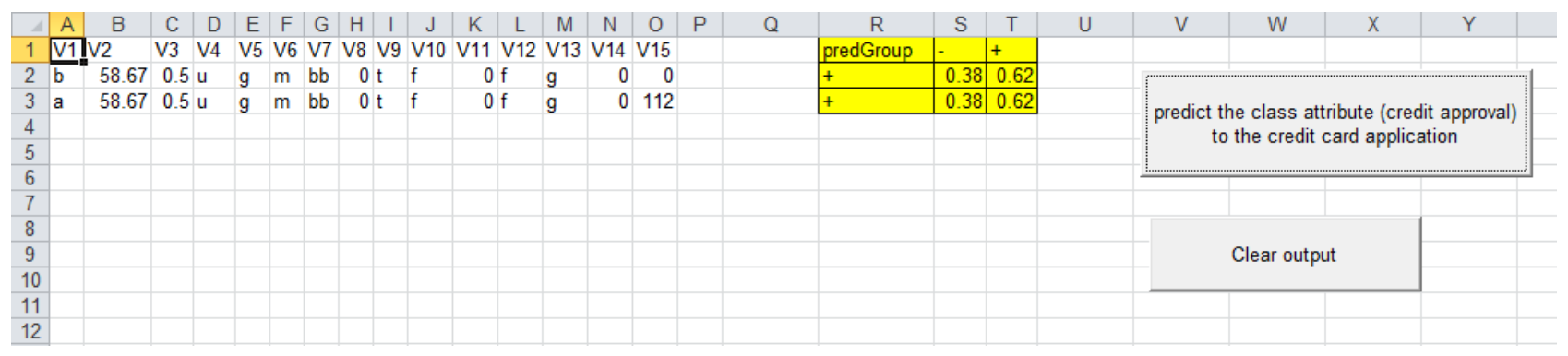

Figure 2: Computation results.

There are a few alternatives to RExcel for connecting Excel with R:

XLLoop provides an Excel Add-In which is able to access various programming languages from within formulae. It supports languages like R, Javascript and others. The "backend" languages are accessed using TCP/IP communication. In contrast to this, RExcel uses COM, which has a very low latency (and hence is very fast). Additionally, the architecture of RExcel supports a completely invisible $\mathrm{R}$ server process (which is what we need for deploying the application), or can provide access to the $\mathrm{R}$ window while developing/testing.

inference for $\mathbf{R}$ provides similar functionality as RExcel. From the website, the latest supported $R$ version is 2.9.1, while $R$ Excel supports current $R$ versions immediately after release (typically new versions work right out of the box without an update in RExcel or statconnDCOM).

\section{Integrating $\mathbf{R}$ and Microsoft Excel}

Work on integration of Excel and $\mathrm{R}$ is ongoing since 1998. Since 2001, a stable environment is available for building Excel documents based upon statistical methods implemented in R. This environment consists of plain R (for Windows) and a toolbox called statconnDCOM with its full integration with Excel using RExcel (which is implemented as an add-in for Microsoft Excel).

Like all Microsoft Office applications and some third-party applications, Excel provides a powerful scripting engine combined with a highly productive visual development environment. This environment is called Visual Basic for Applications, or VBA for short (see Microsoft Corporation (2001)). VBA allows creation of "worksheet-functions" (also called user defined functions or UDFs), which can be used in a similar way as Excel's built-in functions (like, e.g., "SUM" or "MEAN") to dynamically compute values to be shown in spreadsheet cells, and Subs which allow arbitrary computations, putting results into spreadsheet ranges. VBA code can be bundled as an "Add-In" for Microsoft Excel which provides an extension both in the user interface (e.g., new menus or dialogs) and in functionality (additional sheet-functions similar to the built-in sheet functions).

Extensions written in VBA can access third-party components compatible with Microsoft's Component Object Model (COM, see Microsoft Corporation and Digital Equipment Corporation (1995)). The link between R and Excel is built on top of two components: RExcel is an Add-In for Microsoft Excel implementing a spreadsheet-style interface to $\mathrm{R}$ and statconnDCOM. statconnDCOM exposes a COM component to any Windows application which encapsulates R's functionality in an easy-to-use way.

statconnDCOM is built on an extensible design with exchangeable front-end and back-end parts. In the context of this article, the back-end is R. A backend implementation for Scilab (INRIA (1989)) is also available. The front-end component is the COM interface implemented by statconnDCOM. This implementation is used to integrate $\mathrm{R}$ or Scilab into Windows applications. A first version of an Uno (OpenOffice.org (2009)) front-end has already been released for testing and download. Using this front-end $\mathrm{R}$ and 
Scilab can easily be integrated into OpenOffice.org applications, like Calc (see OpenOffice.org (2006)). ROOo is available via Drexel (2009) and already supports Windows, Linux and MacOS X.

RExcel supports various user interaction modes:

Scratchpad and data transfer mode: Menus control data transfer from $\mathrm{R}$ to Excel and back; commands can be executed immediately, either from Excel cells or from $\mathrm{R}$ command line

Macro mode: Macros, invisible to the user, control data transfer and $\mathrm{R}$ command execution

Spreadsheet mode: Formulas in Excel cells control data transfer and command execution, automatic recalculation is controlled by Excel

Throughout the rest of this article, we will describe how to use scratchpad mode and macro mode for prototyping and implementing the application.

For more information on RExcel and statconnDCOM see Baier and Neuwirth (2007).

\section{Implementing the classification and regression tree}

With RExcel's tool set we can start developing our application in Excel immediately. RExcel has a "scratchpad" mode which allows you to write R code directly into a worksheet and to run it from there. Scratchpad mode is the user interaction mode we use when prototyping the application. We will then transform the prototypical code into a "real" application. In practice, the prototyping phase in scratchpad mode will be omitted for simple applications. In an Excel hosted $\mathrm{R}$ application we also want to transfer data between Excel and R, and transfer commands may be embedded in $\mathrm{R}$ code. An extremely simplistic example of code in an $\mathrm{R}$ code scratchpad range in Excel might look like this:

$$
\begin{array}{ll}
\# ! r p u t \quad \text { inval } & \text { 'Sheet1'!A1 } \\
\text { result<-sin(inval) } & \\
\text { \#!rget } \quad \text { result } & \text { 'Sheet1'!A2 }
\end{array}
$$

$\mathrm{R}$ code is run simply by selecting the range containing code and choosing Run R Code from the popup menu.

Lines starting with \# ! are treated as special RExcel commands. rput will send the value (contents) of a cell or range to $\mathrm{R}$, rget will read a value from $\mathrm{R}$ and store it into a cell or range.

In the example, the value stored in cell A1 of sheet Sheet 1 will be stored in the $\mathrm{R}$ variable inval. After evaluating the R expression result<-sin (inval), the value of the $R$ variable result is stored in cell A2.

Table 1 lists all special RExcel commands and provides a short description. More information can be found in the documentation of RExcel.
In the example below, we have a dataset on the credit approval process for 690 subjects. For each record, we have 15 input variables (qualitative and quantitative) while variable 16 indicates the outcome of the credit application: positive $(+)$ or negative $(-)$. Based on this training dataset, we develop our classification tree which is used to show the influence of 15 variables on the loan application and to distinguish "good" applicants from "risky" applicants (so as to estimate variable 16). The risk manager will use this model fully integrated into Excel for further credit approval process.

The goal of the code snippet below is to estimate the model and display the chart with the classification tree. The risk manager can easily view the binary tree and use this chart to highlight the splits. This will help discover the most significant variables for the credit approval process. For example, in this case it is clear that the predictor variables $V 9$ determines the best binary partition in terms of minimizing the "impurity measure". In addition, the risk manager will notice that when $V 9$ equals $a$, the only significant variable to observe is $V 4$. The graphical representation of the classification tree is shown in Figure 3 on page 8.

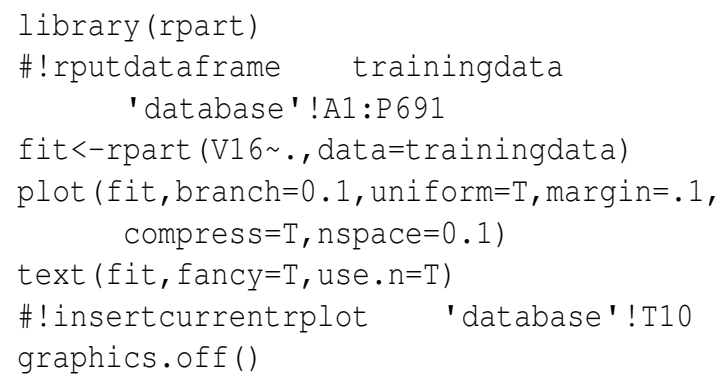

Note: \in the code denotes a line break for readability and should not be used in the real spreadsheet

After estimating the model andvisualizing the results, you can use the Classification Tree to make predictions about the variable V16: the applicants will be classified as "good" or "risky." By running the following code, the 15 observed variables for two people are used to estimate the probability of credit approval. Generally, a "positive" value $(+)$ with probability higher than 0.5 will indicate to grant the loan. So the risk manager using this model will decide to grant the credit in both cases.

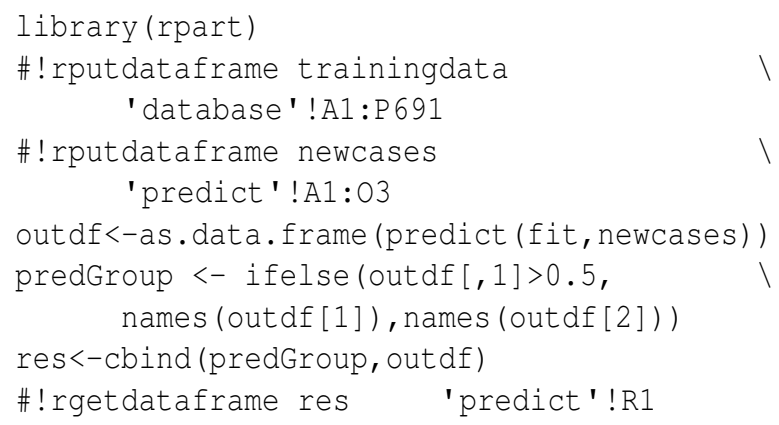




\begin{tabular}{ll}
\hline Command & Description \\
\hline$\# !$ rput variable range & store the value (contents) of a range in an R variable \\
$\# !$ rputdataframe variable range & store the value of a range in an R data frame \\
$\# !$ rputpivottable variable range & store the value of a range in an R variable \\
$\# !$ rget r-expression range & store the value of the R expression in the range \\
$\# !$ rgetdataframe r-expression range & store the data frame value of the R expression in the range \\
$\# !$ insertcurrentplot cell-address & insert the active plot into the worksheet \\
\hline
\end{tabular}

Table 1: Special RExcel commands used in sheets

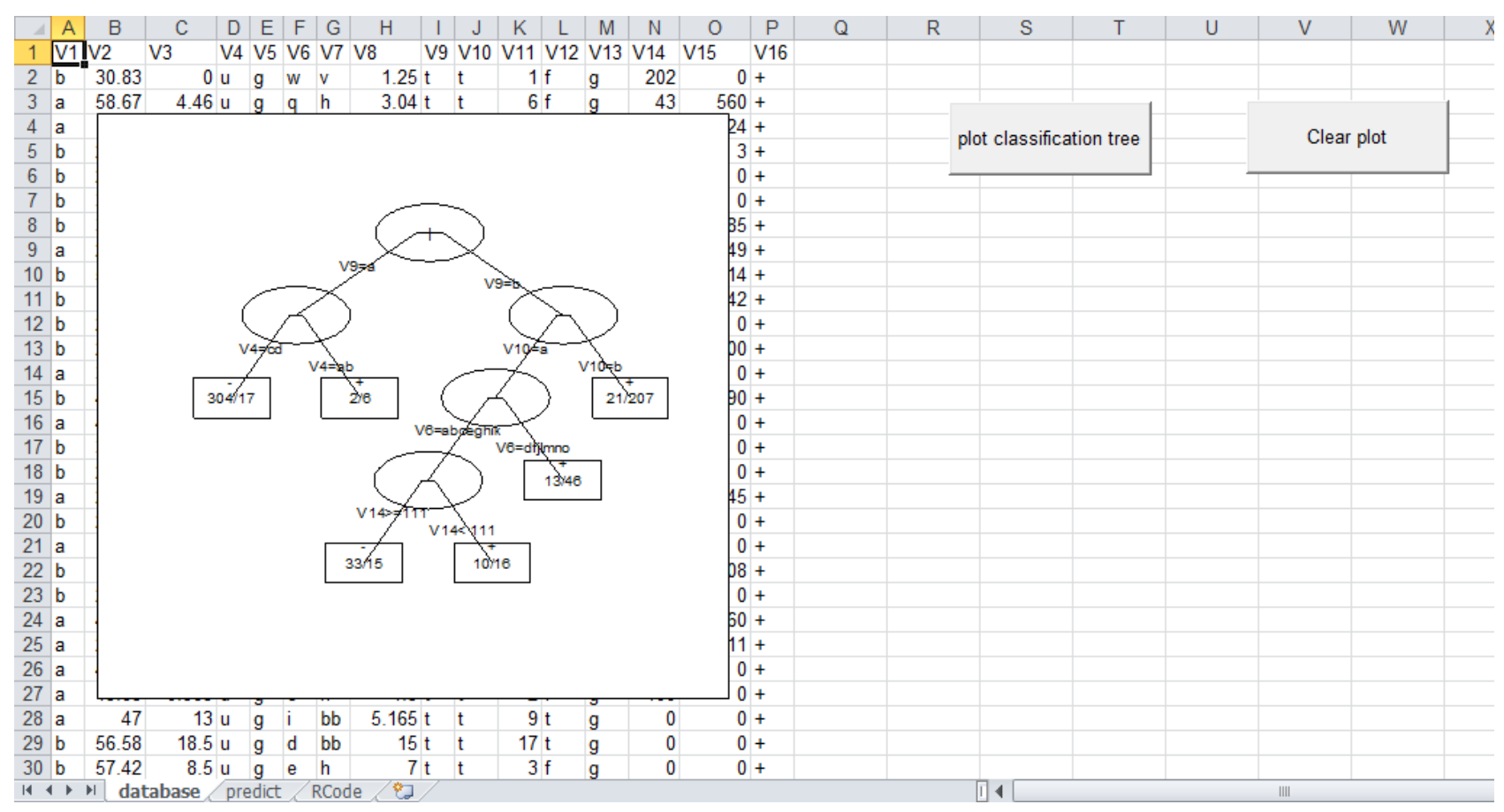

Figure 3: Graphical representation of the classification tree in the RExcel based application. 
The potential of this approach is obvious: Very complex models, such as classification and regression trees, are made available to decision makers (in this case, the risk manager) directly in Microsoft Excel. See Figure 2 on page 6 for a screen-shot of the presentation of the results.

\section{Tidying up the spreadsheet}

In an application to be deployed to end users, $\mathrm{R}$ code should not be visible to the users. Excel's mechanism for performing operations on data is to run macros written in $V B A$ (Visual Basic for Applications, the programming language embedded in Excel). RExcel implements a few functions and subroutines which can be used from VBA. Here is a minimalistic example:

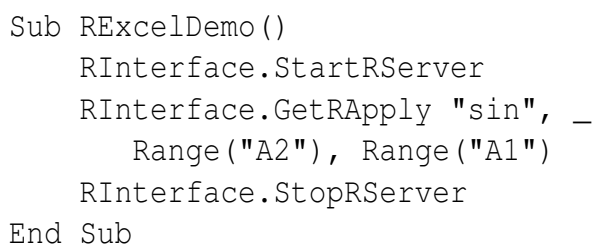

GetRApply applies an R function to arguments taken from Excel ranges and puts the result in a range. These arguments can be scalars, vectors, matrices or dataframes. In the above example, the function sin is applied to the value from cell $\mathrm{A} 1$ and the result is put in cell A2. The $\mathrm{R}$ function given as the first argument to GetRApply does not necessarily have to be a named function, any function expression can be used.

RExcel has several other functions that may be called in VBA macros. RRun runs any code given as string. RPut and RGet transfer matrices, and RPutDataframe and RGetDataframe transfer dataframes. RunRCall similar to GetRApply calls an $\mathrm{R}$ function but does not transfer any return value to Excel. A typical use of RunRCall is for calling plot functions in R. InsertCurrentRPlot embeds the current $\mathrm{R}$ plot into Excel as an image embedded in a worksheet.

In many cases, we need to define one or more $R$ functions to be used with GetRApply or the other VBA support functions and subroutines. RExcel has a mechanism for that. When RExcel connects to R (using the command RInterface.StartRServer), it will check whether the directory containing the active workbook also contains a file named RExcelstart. R. If it finds such a file, it will read its contents and evaluate them with R (source command in R). After doing this, RExcel will check if the active workbook contains a worksheet named RCode. If such a worksheet exists, its contents also will be read and evaluated using $\mathrm{R}$.

Some notes on handling $R$ errors in Excel: The $\mathrm{R}$ implementation should check for all errors which are expected to occur in the application. The application itself is required to pass correctly typed arguments when invoking an R/ function. If RExcel calls a function in $\mathrm{R}$ and $\mathrm{R}$ throws an error, an Excel dialog box will pop up informing the user of the error. An alternative to extensive checking is to use R's try mechanism to catch errors in $\mathrm{R}$ and handle them appropriately.

Using these tools, we now can define a macro performing the actions our scratchpad code did in the previous section. Since we can define auxiliary funcions easily, we can also now make our design more modular.

The workhorse of our application is the following VBA macro:

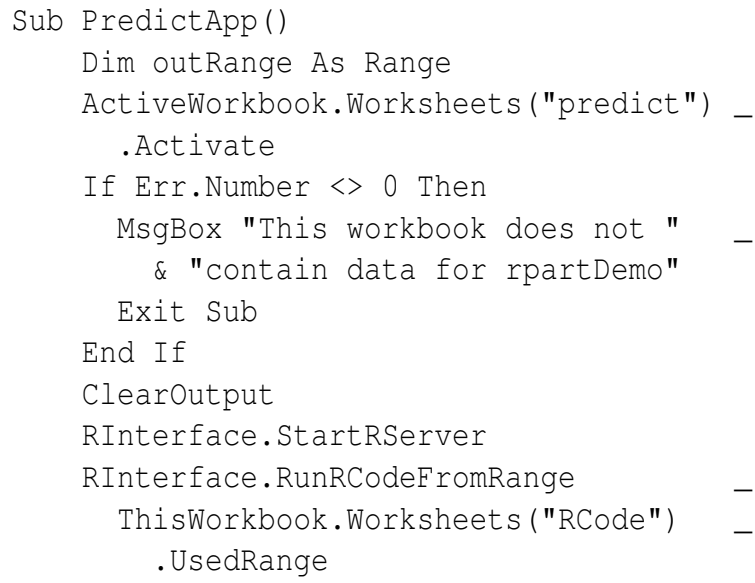

The function predictResult is defined in the worksheet RCode.

\section{Packaging the application}

So far, both our code (the R commands) and data have been part of the same spreadsheet. This may be convenient while developing the RExcel-based application 
or if you are only using the application for yourself, but it has to be changed for redistribution to a wider audience.

We will show how to divide the application into two parts, the first being the Excel part, the second being the $\mathrm{R}$ part. With a clear interface between these, it will be easy to update one of them without affecting the other. This will make testing and updating easier. Separating the R implementation from the Excel (or RExcel) implementation will also allow an R expert to work on the R part and an Excel expert to work on the Excel part.

As an additional benefit, exchanging the Excel front-end with a custom application (e.g., written in a programming language like $\mathrm{C \# )}$ will be easier, too, as the $\mathrm{R}$ implementation will not have to be changed (or tested) in this case.

The application's $\mathrm{R}$ code interface is simple, yet powerful. RExcel will only have to call a single $R$ function called approval. Everything else is hidden from the user (including the training data). approval takes a data frame with the data for the cases to be decided upon as input and returns a data frame containing the group classification and the probabilities for all possible groups. Of course, the return value can also be shown in a figure.

\section{Creating an $R$ package}

The macro-based application built in section "Tidying up the spreadsheet" still contains the $\mathrm{R}$ code and the training data. We will now separate the implementation of the methodology and the user interface by putting all our $\mathrm{R}$ functions and the training data into an $R$ package. This $R$ package will only expose a single function which gets data for the new cases as input and returns the predicted group membership as result. Using this approach, our application now has a clear architecture. The end user workbook contains the following macro:

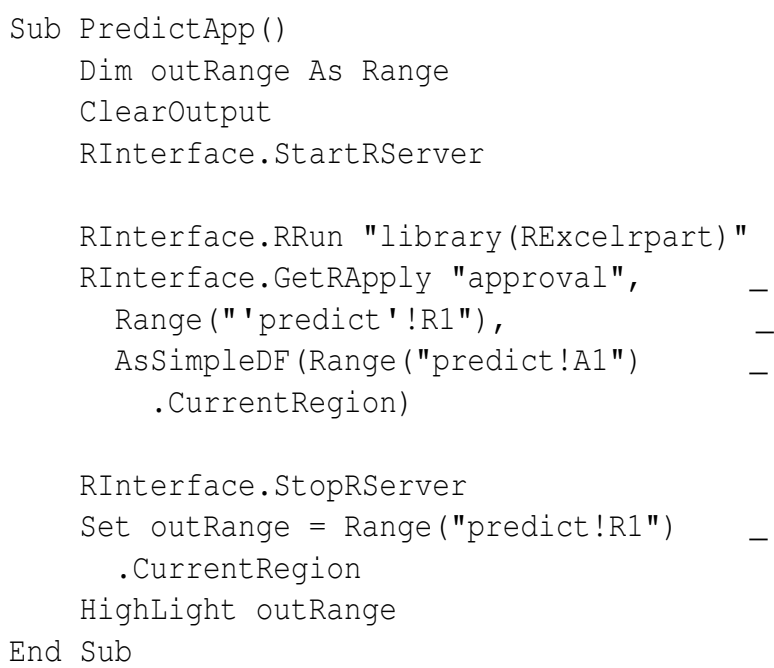

In this macro, we start a connection to $\mathrm{R}$, load the $R$ package and call the function provided by this pack- age and then immediately close the the connection to R.

The statconnDCOM server can reside on another machine than the one where the Excel application is running. The server to be used by RExcel can be configured in a configuration dialog or even from within VBA macros. So with minimal changes, the application created can be turned into an application which uses a remote server. A further advantage of this approach is that the $\mathrm{R}$ functions and data used by the application can be managed centrally on one server. If any changes or updates are necessary, the Excel workbooks installed on the end users' machines do not need to be changed.

\section{Building a VBA add-in for Excel}

The implementation using an R package still has some shortcomings. The end user Excel workbook contains macros, and often IT security policies do to not allow end user workbooks to contain any executable code (macros). Choosing a slightly different approach, we can put all the VBA macro code in an Excel add-in. In the end user workbook, we just place buttons which trigger the macros from the add-in. When opening a new spreadsheet to be used with this add-in, Excel's template mechanism can be used to create the buttons on the new worksheet. The code from the workbook described in section "Tidying up the spreadsheet" cannot be used "as is" since it is written under the assumption that both the training data and the data for the new cases are contained in the same workbook. The necessary changes, however, are minimal. Converting the workbook from section "Tidying up the spreadsheet" again poses the problem that the data and the methodology are now deployed on the end users' machines. Therefore updating implies replacing the add-in on all these machines. Combining the add-in approach with the packaging approach from section "Creating an R package" increases modularization. With this approach we have:

- End user workbooks without any macro code.

- Methodology and base data residing on a centrally maintained server.

- Connection technology for end users installed for all users in one place, not separately for each user.

\section{Deploying the Application}

Using the application on a computer requires installation and configuration of various components.

The required (major) components are:

- Microsoft Excel, including RExcel and statconnDCOM

- $\mathrm{R}$, including rscproxy 
- The VBA Add-In and the R package created throughout this article

For the simplest setup, all compontents are installed locally. As an alternative, you can also install Excel, RExcel and our newly built VBA Add-In on every workstation locally and install everything else on a (centralized) server machine. In this case, $R$, rscproxy, our application's $R$ package and statconnDCOM are installed on the server machine and one has to configure RExcel to use $\mathrm{R}$ via statconnDCOM on a remote server machine. Please beware that this kind of setup can be a bit tricky, as it requires a correct DCOM security setup (using the Windows tool dcomcnfg).

\section{Downloading}

All examples are available for download from the Download page on http://rcom. univie.ac.at.

\section{Bibliography}

E. I. Altman. Bankruptcy, Credit Risk, and High Yield Junk Bonds. Blackwell Publishers Inc., 2002.

T. Baier. rcom: R COM Client Interface and internal COM Server, 2007. R package version 1.5-1.

T. Baier and E. Neuwirth. R (D)COM Server V2.00, 2005. URL http://cran.r-project.org/other/ DCOM.

T. Baier and E. Neuwirth. Excel :: COM :: R. Computational Statistics, 22(1):91-108, April 2007. URL http://www.springerlink.com/content/ uv6667814108258m/.

Basel Committee on Banking Supervision. International Convergence of Capital Measurement and Capital Standards. Technical report, Bank for International Settlements, June 2006. URL http: //www.bis.org/publ/bcbs128.pdf.

L. Breiman, J. Friedman, R. Olshen, and C. Stone. Classification and Regression Trees. Wadsworth and Brooks, Monterey, CA, 1984.

J. M. Chambers. Programming with Data. Springer, New York, 1998. URL http://cm.bell-labs. $\mathrm{com} / \mathrm{cm} / \mathrm{ms} /$ departments/sia/Sbook/. ISBN 0-38798503-4.

R. Drexel. ROOo, 2009. URL http: //rcom.univie.ac . at/.
T. Hastie, R. Tibshirani, and J. Friedman. The Elements of Statistical Learning: Data Mining, Inference, and Prediction. Springer, 2009.

INRIA. Scilab. INRIA ENPC, 1989. URL http: //www. scilab.org.

B. D. McCullough and B. Wilson. On the accuracy of statistical procedures in Microsoft Excel 2000 and Excel XP. Computational Statistics and Data Analysis, 40:713-721, 2002.

Microsoft Corporation. Microsoft Office 2000/Visual Basic Programmer's Guide. In MSDN Library, volume Office 2000 Documentation. Microsoft Corporation, October 2001. URL http: //msdn.microsoft.com/.

Microsoft Corporation and Digital Equipment Corporation. The component object model specification. Technical Report 0.9, Microsoft Corporation, October 1995. Draft.

OpenOffice.org. OpenOffice, 2006. URL http: //www . openoffice.org/.

OpenOffice.org. Uno, 2009. URL http://wiki. services.openoffice.org/wiki/Uno/.

H. Zhang and B. Singer. Recursive Partitioning in the Health Sciences. Springer-Verlag, New York, 1999. ISBN 0-387-98671-5.

Thomas Baier

Department of Scientific Computing

Universitity of Vienna

1010 Vienna

Austria

thomas.baier@univie.ac.at

Erich Neuwirth

Department of Scientific Computing

Universitity of Vienna

1010 Vienna

Austria

erich.neuwirth@univie.ac.at

Michele De Meo

Venere Net Spa

Via della Camilluccia, 693

00135 Roma

Italy

micheledemeo@gmail.com 